\section{In vivo assessment of three dimensional coronary anatomy using electron beam computed tomography after intravenous contrast administration}

\author{
B J Rensing, A H H Bongaerts, R J van Geuns, P M A van Ooijen, M Oudkerk, P J de Feyter
}

Correspondence to: Dr Rensing.

Accepted for publication 15 June 1999

\begin{abstract}
Department of
Cardiology,

Thoraxcenter BD 416,

Erasmus University

Rotterdam, Dr

Molewaterplein 40,

3015 GD Rotterdam,

Netherlands

B J Rensing

R J van Geuns

P M A van Ooijen

P J de Feyter

Department of Radiology, Dr Daniel den Hoed Kliniek,

Rotterdam,

Netherlands

A H H Bongaerts

M Oudkerk

Abstract

Intravenous coronary angiography with electron beam computed tomography (EBCT) allows for the non-invasive visualisation of coronary arteries. With dedicated computer hardware and software, three dimensional renderings of the coronary arteries can be constructed, starting from the individual transaxial tomograms. This article describes image acquisition, postprocessing techniques, and the results of clinical studies. EBCT coronary angiography is a promising coronary artery imaging technique. Currently it is a reasonably robust technique for the visualisation and assessment of the left main and left anterior descending coronary artery. The right and circumflex coronary arteries can be visualised less consistently. Improvements in image acquisition and postprocessing techniques are expected to improve visualisation and diagnostic accuracy of the technique.

(Heart 1999;82:523-525)
\end{abstract}

Keywords: electron beam computed tomography; coronary angiography; coronary anatomy; volume rendering
Recently intravenous coronary angiography using electron beam computed tomography (EBCT) has emerged as a new coronary imaging technique. ${ }^{1-7}$ By virtue of the absence of moving parts, EBCT can construct ECG triggered, high resolution tomograms in $100 \mathrm{~ms}$. This is fast enough to reduce cardiac motion artefacts and produce high quality cardiac images, ${ }^{8}$ especially when the acquisition window is set during diastasis. The scanner was originally designed in the 1980s for cardiac function studies and myocardial perfusion imaging. ${ }^{8}$ Later it was applied for the accurate quantification of coronary calcification.

\section{Technique}

IMAGE ACQUISITION

Scanning is performed with the patient in the supine position. Contrast (iopromide $350 \mathrm{mg} /$ $\mathrm{ml}$ ) is injected through an antecubital vein to a total of $150 \mathrm{ml}$. Tomography starts just proximal to the left main coronary artery after an ECG trigger at $80 \%$ of the RR interval (diastasis). A tomogram thickness of $1.5 \mathrm{~mm}$ and a table increment after each tomogram of $1.5 \mathrm{~mm}$ results in contiguous non-overlapping slices. A total of 40-60 contrast enhanced tomograms are made during a single breath hold after intravenous contrast administration
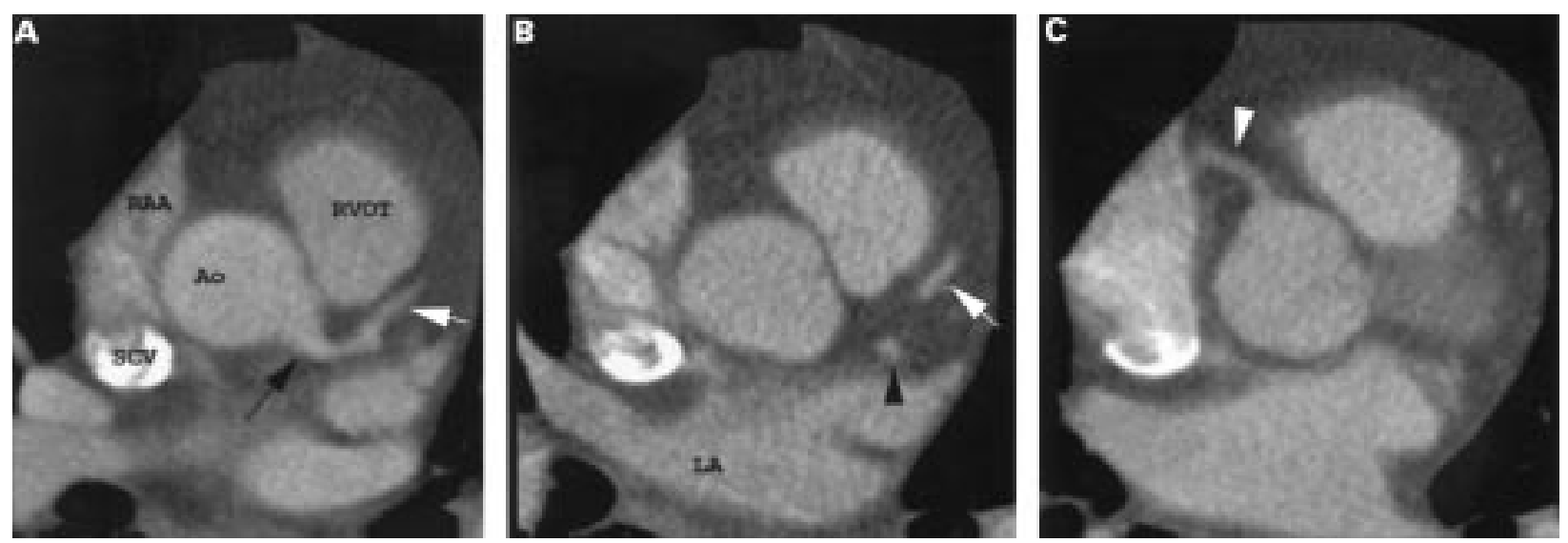

Figure 1 (A) Transaxial tomogram after intravenous contrast injection. Ao, ascending aorta; RVOT, right ventricular outflow tract, $R A$ A, right atrial appendage; black arrow, left main coronary artery; white arrow, proximal left anterior descending artery. (B) Transaxial tomogram at a lower level. LA, left atrium; black arrowhead, proximal circumflex artery cut perpendicular. (C) Transaxial tomogram at the level of origin of the right coronary artery (white arrowhead). 

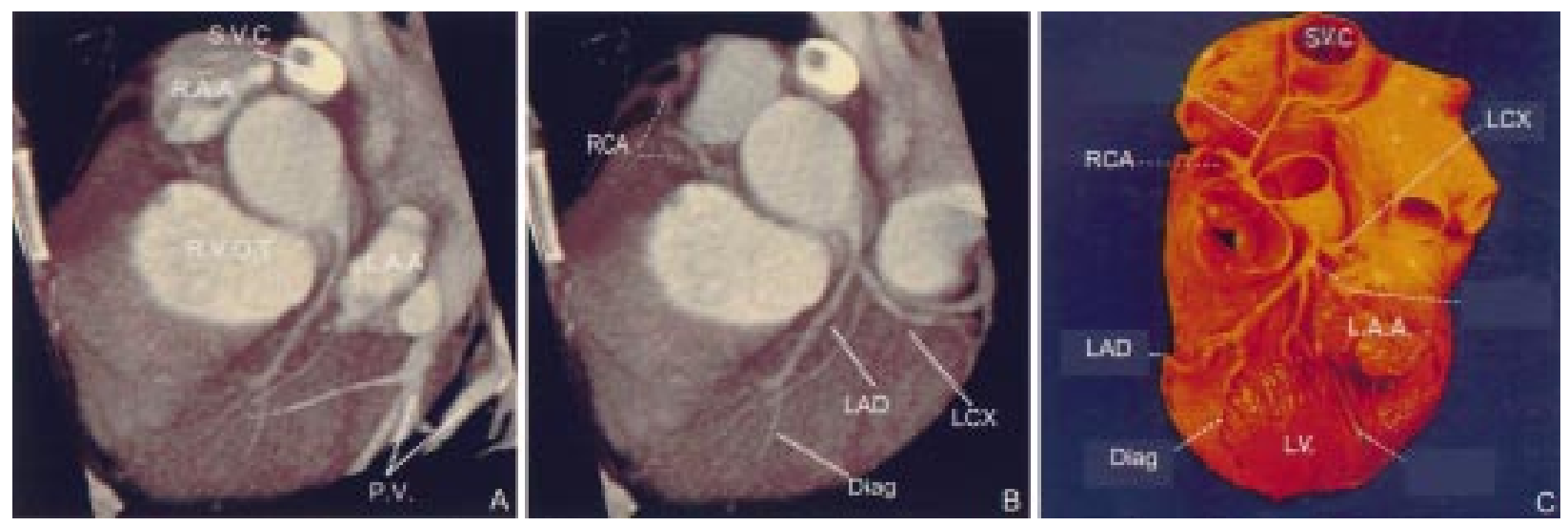

Figure 2 Rendering of the left coronary artery before $(A)$ and after (B) removal of the left atrial appendage (LAA). In (A) the left circumflex coronary artery (LCX) is hidden under the left atrial appendage. At the tomographic level the atrial appendage was removed manually on 15 levels before rendering (B) was made. (C) Pressure fixed anatomical specimen showing the relation between atrial appendage and circumflex artery. Diag, ramus diagonalis; $L A D$, left anterior descending coronary artery; PV, pulmonary veins; $R A A$, right atrial appendage; RCA, right coronary artery; RVOT, right ventricular outflow tract; SVC, superior vena cava. Reproduced from McAlpine ${ }^{13}$ with permission of Springer Verlag.

(fig 1). Radiation exposure for a complete examination is estimated to be $<20 \mathrm{mGy}^{3}{ }^{9}$ This is approximately one fifth of the radiation exposure at coronary angiography. ${ }^{10}$ It is our $^{3}$ and others ${ }^{11}$ experience that after instruction most patients are able to hold their breath for at least 35 seconds.

The individual tomograms have a resolution of 4-6 linepairs $/ \mathrm{cm}$ and the scanning time for each tomogram is $100 \mathrm{~ms}$. The spatial resolution in the scanning direction is limited by the slice thickness of $1.5 \mathrm{~mm}$. Very short lesions $(<3 \mathrm{~mm}$ long) in artery segments that are perpendicular to the scanning plane can therefore be missed. Although these specifications fall short of the spatial (5 linepairs $/ \mathrm{mm}$ ) and temporal resolution (up to 50 frames/s) of conventional cine coronary angiography, proximal and middle parts of the coronary arteries can be visualised with EBCT.

THREE DIMENSIONAL RECONSTRUCTION

Two dimensional transaxial tomograms are transferred to a computer workstation where they are stacked and interpolated to form a three dimensional volume using specialised software. This process creates a database representation of a three dimensional object. With special rendering techniques this database representation of the three dimensional object can be visualised. Several three dimensional rendering techniques are available, ${ }^{12}$ but we almost exclusively use volume rendering. ${ }^{3}$

Volume rendering allows certain parts of the three dimensional object to be transparent so that structures behind these parts are still visible. By assigning the right colour and opacity values to different tissues, real anatomy is simulated in much the same way as pictures in an anatomic atlas (figs 2,3 , and 4$).{ }^{13}$

\section{Clinical performance}

Several groups have compared intravenous EBCT coronary angiography to conventional angiography. ${ }^{2-7}$ In all these studies both the original tomographic data and the three dimensional renderings were used to assess the coronary arteries. Overall, the technique

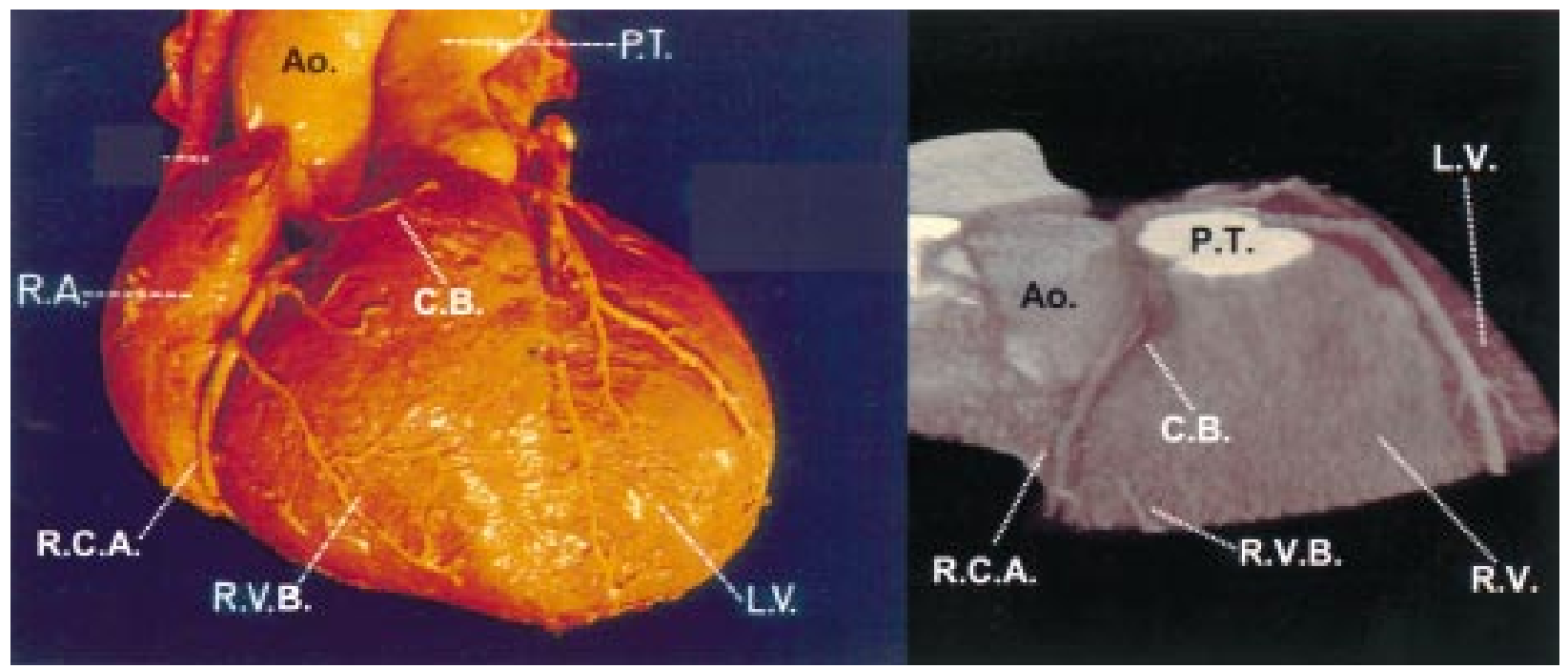

Figure 3 (Left) A pressure fixed anatomical specimen showing the proximal and middle right coronary artery (RCA) with its side branches (conus branch $(C B)$ and right ventricular branch (RVB)). Reproduced from McAlpine ${ }^{13}$ with permission of Springer Verlag. (Right) A three dimensional rendering of the right coronary artery. Because of the small size, only the proximal part of the conus branch could be seen. The right ventricular branch has a stenosis in the proximal part. At the right side of the picture the left anterior descending coronary artery can be clearly seen. Ao, ascending aorta; PT, pulmonary trunk; $L V$, left ventricle; $R V$, right ventricle; $R A$, right atrium. 

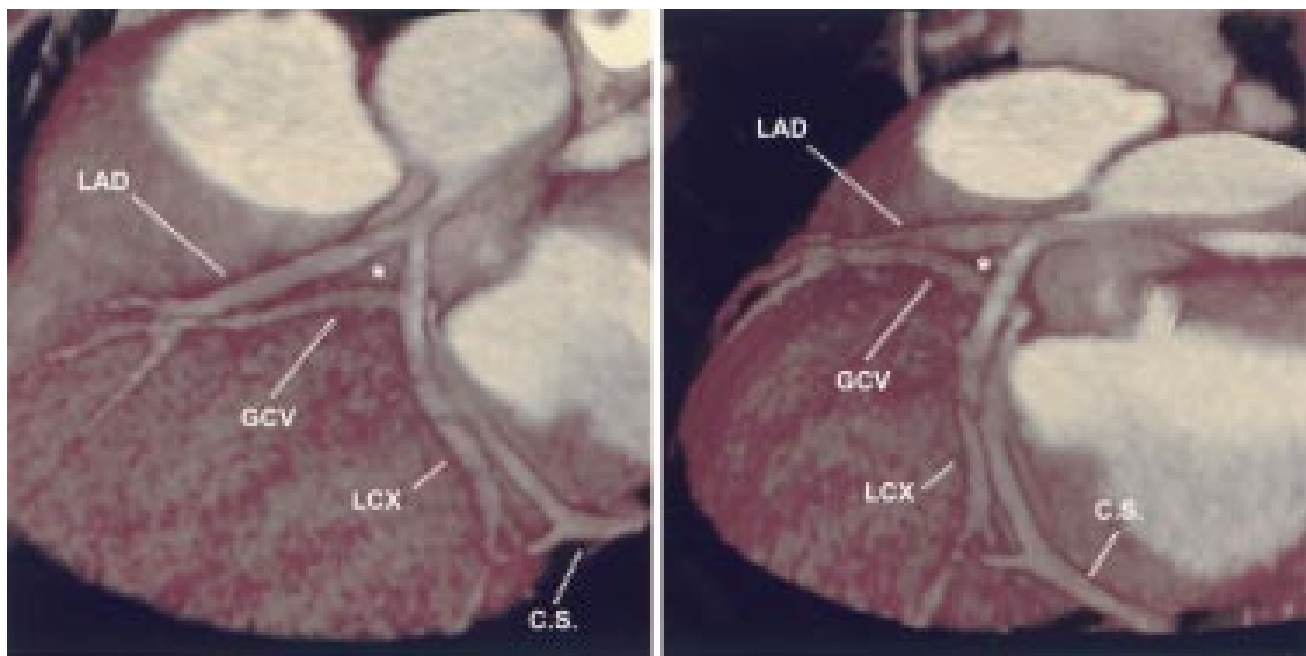

Figure 4 Renderings of the left coronary arterial and venous systems. (Left) Great cardiac vein (GVC) running parallel to the left anterior descending coronary artery (LAD), crossing under the circumflex artery (LCX) and entering the coronary sinus (CS). ${ }^{\star}$ Triangle of Brocq and Mouchet formed by the proximal left anterior descending coronary artery, the proximal circumflex coronary artery, and the great cardiac vein crossing from the anterior interventricular groove to the atrioventricular groove. (Right) Three dimensional rendering of the same dataset offering a more lateral and posterior view of the heart, arteries, and veins. This clearly shows the possibility of the three dimensional rendering technique to view the object from any angle.

allowed visualisation of the proximal and midcoronary arteries in approximately $80 \%$ of cases. The left main coronary artery and proximal and middle parts of the left anterior descending coronary artery could generally be visualised and assessed in $90-100 \%$ of cases, with a sensitivity to detect a significant stenosis of $85-90 \%$ and a specificity of around $90 \%$. Images of the right and circumflex coronary arteries were interpretable in only $75 \%$ of cases, with a little lower diagnostic accuracy in general. Major causes for non-assessability were cardiac motion artefacts, and the smaller size of the middle and distal parts of the right and circumflex arteries. Improvements in ECG triggering, shorter acquisition time (50 ms high resolution tomograms), and improved spatial resolution with a recently introduced new detector ring are expected to improve visualisation and diagnostic accuracy of the technique.

CONCLUSION

Intravenous EBCT coronary angiography is a promising imaging modality that allows for non-invasive visualisation of the proximal and middle parts of the coronary arteries.

1 Moshage WE, Achenbach S, Seese B, et al. Coronary artery stenoses: three dimensional imaging with electrocardiographically triggered, contrast enhanced, electron beam CT. Radiology 1995;196:707-14.
2 Schmermund A, Rensing BJ, Sheedy PF, et al. Intravenous electron beam CT coronary angiography for segmental analysis of significant coronary artery stenoses: feasibility and limitations. $7 \mathrm{Am}$ Coll Cardiol 1998;31:1547-54.

3 Rensing BJ, Bongaerts A, van Geuns RJ, et al. Intravenous coronary angiography by electron beam computed tomography. A clinical evaluation. Circulation 1998;98:2509-12.

4 Achenbach S, Moshage W, Ropers D, et al. Value of electron-beam computed tomography for the noninvasive detection of high-grade coronary-artery stenoses and occlusions. N Engl f Med 1998;339:1964-71.

5 Nakanishi T, Ito K, Imazu M, et al. Evaluation of coronary artery stenoses using electron-beam CT and multiplanar reformation. f Comput Assist Tomogr 1997;21:121-7.

6 Reddy PR, Chernoff DM, Adams JR, et al. Coronary artery stenoses: assessment with contrast enhanced electron beam CT and axial reconstructions. Radiology 1998;208:167-72.

7 Budoff MJ, Oudiz RJ, Zalace CP et al. Intravenous three-dimensional coronary angiography using contrast enhanced electron beam computed tomography. Am f Cardiol 1999;83:840-5.

8 Stanford W, Rumberger JA. Ultrafast computed tomography in cardiac imaging: principles and practice. Mount Kisco, New York: Futura, 1993:1-16.

9 Wexler L, Brundage B, Crouse, J, et al. Coronary artery calcification: pathology, epidemiology, imaging methods, and clinical implications. A statement for health professionals from the American Heart Association. Circulation 1996; 94:1175-92.

10 Stehling MK, von Smekal A, Reise M. MRI der Koronararterieen Moglichkeiten und Grenzen. Radiologe 1994;34: 462-8.

11 Gay SB, Sistrom CL, Holder CA, et al. Breath-holding capability of adults. Implications for spiral computed tomography, fast acquisition magnetic resonance imaging, and angiography. Invest Radiol 1994;29:848-51.

12 Van Ooyen PMA, de Feyter PJ, Oudkerk M. An introduction to three dimensional cardiac image rendering and processing. Cardiologie 1997;4:312-19.

13 McAlpine WA. Heart and coronary arteries. Heidelberg: Springer Verlag, 1975. 